\title{
'Compliance in Chronic Obstructive Pulmonary Disease Patients Attending Pulmonary Medicine OPD in a Tertiary Care Hospital: Prospective study'
}

\author{
Dr. Anshu Gupta ${ }^{1,}$ Dr. Dinesh Mehta ${ }^{2,}$ Dr. Sulbha Singla ${ }^{3,}$ Dr. Kusum Singla ${ }^{4}$ \\ 1. Assistant professor. Dept. of Pharmacology, MMIMSR, Mullana, Ambala, Haryana, India. \\ 2. Assistant Professor Dept. of Pulmonary medicine, MMIMSR, Mullana, Ambala, Haryana, India. \\ 3. Post Graduate Student, Dept. of Pulmonary medicine, MMIMSR, Mullana, Ambala, Haryana, India. \\ 4. Post Graduate Student, Dept. of Biochemistry, MMIMSR, Mullana, Ambala, Haryana, India
}

\begin{abstract}
Non Compliance in patients with Chronic Obstructive Pulmonary Disease (COPD) to the medication can result in worsening of the disease and increases the hospital admissions. This study aimed at examining the dimensions of compliance to the medication, life style modification and smoking cessation in 60 COPD patients. A pre validated questionnaire was administered at 0 week, 2 weeks and at 6 weeks. Ninety percent of the patients took the prescribed oral drugs in correct dose, correct timings and 77\% complied to hospital visits. In contrast to oral drugs only $36 \%$ of the patients were compliant to the use of inhaler at the first visit which improved to 59\%. Life style changes related to diet and exercise were followed by 32\% patients. Only $19 \%$ of patients quit smoking. In order to improve compliance levels repeated counseling or stressing the importance of use of inhalers and quitting smoking was found an effective method.
\end{abstract}

Key Words: Chronic Obstructive Pulmonary Disease (COPD), Compliance, Smoking cessation, Inhalers.

\section{Introduction}

Chronic Obstructive Pulmonary Disease (COPD) is characterized by an irreversible decline in lung function, exercise capacity and health status. ${ }^{1}$ COPD is a chronic illness and poor adherence of patients to the disease management may result in increased rate of morbidity, health care expenditures, hospitalizations and reduced Quality of Life (QoL). There can be increase in the dose of therapy. It can be affected by the various perceptions of the patient about the disease, social beliefs, type of treatment and medication, quality of service provider and social environment. ${ }^{2}$

Many of times there is acute clinical worsening of disease and it may necessitate a change in regular therapy. Majority of exacerbations are not reported and most likely not treated. These events have clinically relevant impact on Health Related Quality of Life (HRQoL). ${ }^{1}$

International guidelines for the treatment of COPD recommend therapy with one or more bronchodilators in patients with moderate disease, and addition of an inhaled corticosteroid in patients with severe or very severe COPD. Bronchodilators which are long acting are preferred over the short acting agents. ${ }^{3}$

According to Jing Jin et al. compliance is the extent to which the behavior of the patient corresponds in terms of taking medication, following diet instructions or executing life style changes with agreed recommendations of from a health care provider. ${ }^{4}$ It is often defined as the extent to which a person behavior coincides with the clinical prescription.

There are various compliance factors i.e. Patient related factors, regimen related factors and health care provider related factors. ${ }^{2,4}$ In this study the patients were prescribed bronchodilators, anticholinergics, and inhaled corticosteroids. Drugs were prescribed according to the severity of disease. Several studies have shown that combination of long acting B-agonist with an inhaled corticosteroid offered additional bronchodilator efficacy over a bronchodilator alone. ${ }^{5}$

In this study we are taking into consideration the patient related factors including the demographic factors such as age, sex, marital status, ethnicity, severity of the disease.

Compliance factors: knowledge about the disease, regimen and benefits and beliefs about the disease.

Assessment of the disease was done by the physical examination, chest X-ray, blood biochemistry and spirometry. FEV1 and FEV1/ FVC ratio was done on each patient on the first visit. 


\section{Material and Methods}

This was a tertiary care hospital based open labeled questionnaire based 6 months study from March 2011 till end of Aug 2011. The objective of the study was to assess the compliance of COPD patients. The present study was conducted after taking Institutional Ethics Committee approval and a written informed consent from participating patients. It aimed at examining the dimensions of compliance in COPD patients to the:

\section{- Medication}

- life style

- smoking cessation

The data of total of 60 patients was collected who met the inclusion criteria, gave the written consent to participate and completed the study. Inclusion criteria were age more than equal to 18 yrs. Symptoms of dyspnoea and chronic cough with or without sputum production. History of smoke exposure, a post-bronchodilator FEV1/FVC of < equal0.7 according to Global Initiative for Chronic Obstructive Lung Disease (GOLD) standards. Patients with asthma, cardiac diseases or other major functionally limiting disease were excluded. A pre validated questionnaire comprising of 15 questions was administered at 0 week, 2 weeks and at 6 weeks to assess the compliance of patients to the medication, life style modification and to quit smoking in smokers. The questionnaire was pre validated on 10 patients and then administered after making the required changes. The diagnosed patients were asked to fill the questionnaire at zero weeks. They were given all the instructions regarding the medication dose frequency and timings. They were also instructed for the use of inhaler, the technique ${ }^{()}$of using inhaler was taught to them to ensure proper drug inhalation by the patient. All the patients were reinforced for smoking cessation. They were shown the pamphlets or charts how smoking continuation can worsen their diseases and quality of life. Instructions regarding life style changes and diet were also given to the patients.

The questionnaire included both open and closed ended questions with study measures:

\section{Clinical and demographic variables}

Demographic and clinical data collected was related to compliance of patients. These factors consist of demographic variables (age, gender, education, ethnicity, profession, urban or rural population), and clinical variables such as (history of smoking, duration of COPD, previous hospitalization).

\section{COPD Compliance Questionnaire}

To assess the compliance of COPD patients following parameters were recorded

Medication both dose and timing, appointment keeping, smoking cessation, exposure to smoke and exercise. Subsequently in the next visits they were asked whether they had difficulty in complying with the various parameters.

\section{Knowledge and beliefs:}

The knowledge regarding their disease, smoking habits and its relation with the COPD and its progression was also recorded. The beliefs regarding medication, inhalers and the various barriers about their compliance were asked.

Consort Statement of the study (Figure 1)

\section{Results:}

Clinical and demographic data was assessed on 60 patients (Table 1). Sixty five percent of the patients were in the age group of 51 to 70 years. Eighty eight percent of the patients were males and rest females. Eighty percent of the patients were of rural origin. Patients working on chullah, and occupations such as chimney workers and brick kiln workers were also associated more with suffering from COPD. They had symptomatic relief at 6 weeks on following the instructions, and decreasing the chullah exposure by female patients. Eight patients with the illness more than 5 years had been hospitalized previously once (3\%) and twice $(8.3 \%)$ for exacerbation of their disease. 
Table 1: Demographic and Clinical Variables in Patients of COPD

\begin{tabular}{|l|l|l|}
\hline \multicolumn{2}{|l|}{ Demographic Factors } & No of Patients (\%) \\
\hline Age (years) & $<30$ & $2(3.3)$ \\
& $31>50$ & $11(18.3)$ \\
& $51>70$ & $39(65)$ \\
& 71 and above & $(13.4)$ \\
\hline Gender & Males & $53(88.3)$ \\
& Females & $7(11.7)$ \\
\hline Area & Urban & $12(20)$ \\
& Rural & $48(80)$ \\
\hline Smoking & Active Smokers & $39(65)$ \\
& Non smokers & $9(15)$ \\
& Passive smokers (ciggerette, chimneys, chullah) brick kiln & $12(20)$ \\
\hline Income per month (Rs) & workers & $31(51.6)$ \\
& Low (<3000) & $24(40)$ \\
\hline Education & Middle (3000-10000) & $5(8.3)$ \\
\hline Duration of disease & High (> 10000) & $46(76.7)$ \\
& Literates & $14(23.3)$ \\
\hline Any & Llliterates & $34(56.7)$ \\
acute attack & More than five years & $26(43.3)$ \\
\hline
\end{tabular}

Overall compliance of the patients to the use of medication was $74 \%$. Ninety percent of the patients took the prescribed oral drugs in correct dose, correct timings at the second visit at $2^{\text {nd }}$ week which improved to $94.3 \%$ by the end of 6 weeks. Seventy seven percent of patients visited the hospital the same day as being told at second week which improved to $82 \%$ by $3^{\text {rd }}$ visit. Ten percent of patients could not visit the same day as being advised. The causes for their non compliance were: their work, could not get the leave the same day, were busy at home or had no conveyance. Patients had improvement of their symptoms over a period of time. Only 2 patients had exacerbation of the disease during study period and were hospitalized for 4-5 days. They were nebulized and had injectable drugs. The symptoms improved and they were compliant to their medication and lifestyle afterwards.

Only $36 \%$ of the patients were compliant to the use of inhaler at the first visit which improved to $59 \%$ with time. (FigureII) Seven percent of the rural and uneducated patients believed that use of inhalers could be habit forming, so they were counseled against their false beliefs. Literate patients were aware about the health hazards of smoking, 10 patients admitted that their disease was due to and is aggravated by smoking. Sixty five percent of the patients wanted to quit the smoking and only 19\% of them quitted at the end of the study (Figure III). Strategy for smoking cessation included counseling and nicotine gum whenever they had a craving for smoking. Life style changes related to diet and exercise were followed by $32 \%$ (Figure IV).

\section{Discussion}

COPD has been recognized as a disease which results in increased morbidity, mortality and and a compromised quality of life in later stages. Usually the patients have daily chronic symptoms, incomplete relief from the disease and progressive deterioration. Treatment with multiple drugs, lifestyle modification and adherance to the drug therapy plays a crucial role to prevent and control the exacerbations. ${ }^{6}$ This study shows that patient adherence to medication is low due to incomplete relief from the symptoms, acute exacerbations on exposure to dust and lack of smoking cessation. Compliance with medication also varies with the level of education. The educated patients were able to understand the importance of medication, correct dosing and time.

In TORCH study, ${ }^{7}$ it was concluded that though the association between increased adherence, improved mortality and reduction in hospital admission was independent of treatment given to patients. But at the same time the effect of treatment was more pronounced in patients with good adherence than in those with poor adherence. Even adherence to inhaled medication was significantly associated with reduced risk of death and admission to hospital due to exacerbations in COPD.

According to Bourbeau $\mathbf{J}$ and Bartlett SJ physicians also play a critical role in helping patients understand the nature of the disease, potential benefits of treatment, encouraging patients to develop self-management skills and addressing concerns regarding potential adverse effects and events. ${ }^{8}$ It is important to explore patients beliefs and concerns about the safety and benefits of the treatment, as patients may have their own fears. The treatment should be kept simple as complex regimens and polytherapy also contribute to suboptimal adherence. ${ }^{8}$ 
In this study majority of the patients were given information about lifestyle change and ongoing management. They were also taught the technique of effective use of the inhaler. But the information received was not that effective as the patients have their own beliefs and due to their low education level and certain myths about the use of inhalers.

It has been found in many studies that physicians should also adhere to a written action plan for the treatment of acute exacerbation of COPD. As it is helpful in decreasing the recovery time and hospital stay of the patients. ${ }^{9}$

Figure 1

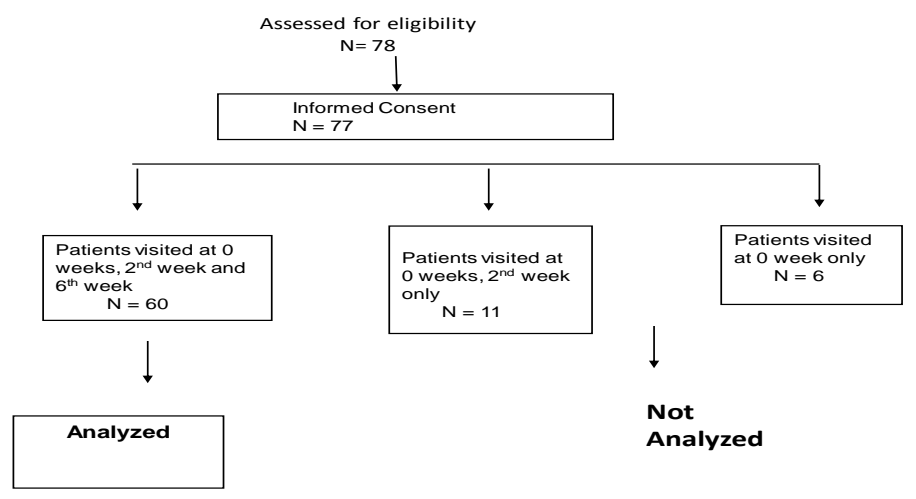

Figure II

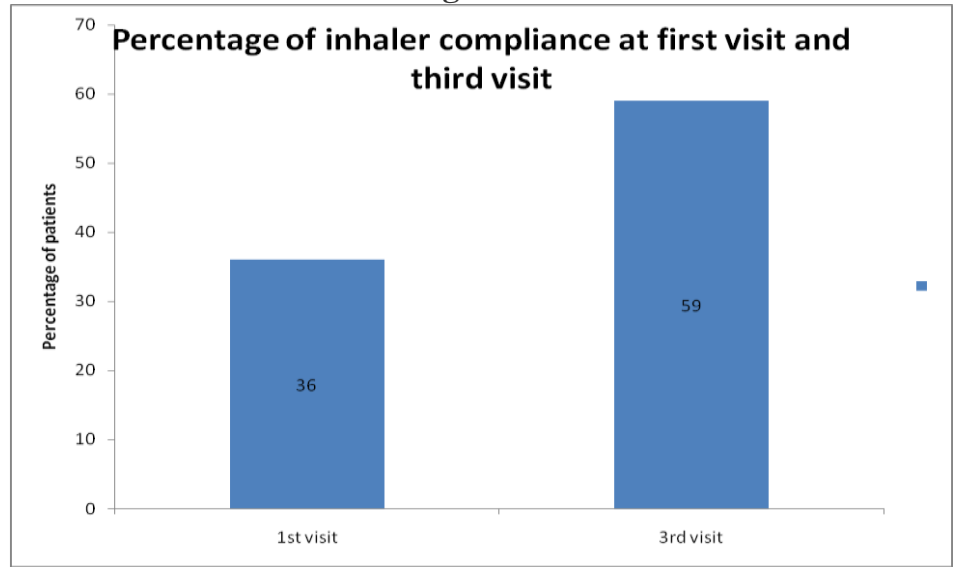

Figure III

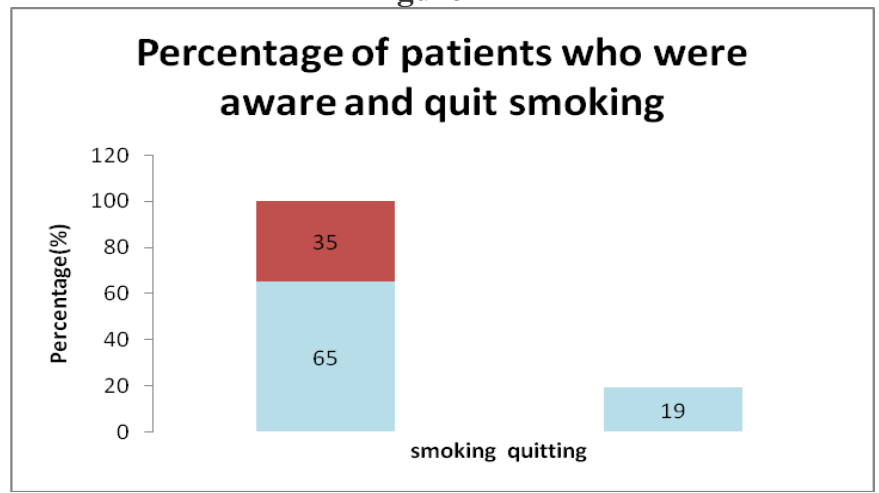


Figure IV

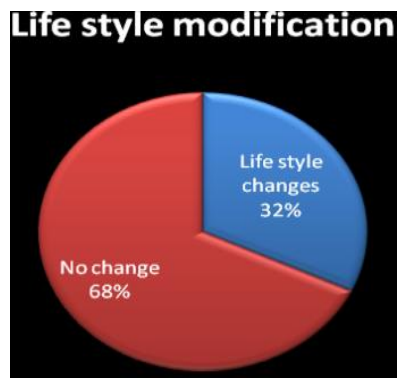

V. Conclusions

The present study represents the compliance of COPD patients to the medication, dose and duration, lifestyle changes. The data indicates that elderly, male patients of rural origin are less compliant as compared to that of urban, educated people. Patients with low level of education and low income are less compliant to the instructions given to them. Most of the patients are compliant with the instructions regarding inhaler use, correct method and dose. It helped them to relieve their symptoms and decreased the acute exacerbation of the disease. Counseling regarding smoking cessation was helpful in some patients. This study concludes that the physicians should counsel the patient about their disease, emphasize on the medication to be taken in correct dose, timings and method of inhaler use to improve the quality of life of patients of COPD

\section{References}

[1]. Trappenburg J CA, Touwen I, Bourbeau J, Monninkhof EM, Verheij T JM, Lammers J W J et al. Detecting exacerbations using the Clinical COPD QuestionnaireHealth and quality of life outcomes 2010; 8: 102.

[2]. Vander Wall MHL, Jaarsmal T, Veeger JGM N, Gilst WHV, Veldhuisen DJV. Compliance in heart failure patients: the importance of knowledge and beliefs. Eur heart J 2005; 27: 434-40.

[3]. Rabe KF, Timmer W, Sagkriotis A, Viel K. Comparison of a Combination of Tiotropium Plus Formetrol to Salmetrol Plus Fluticasone in Moderate COPD. Chest 2008; 134: 255-63.

[4]. Jin J, Sklar GE, Oh VMS, Li Shu Chuen. Factors affecting therapeutic compliance: A review from the patient's perspective. Ther Clin Risk Manag 2008 ; 4: 269-86.

[5]. . Beauchamp MK, Nonoyama M, Goldstein RS, Hill K, Dolmage TE, Mathur S,Brooks D. Interval versus continuous training in individuals with chronic obstructive pulmonary disease- a systematic review. Thorax 2010; 65: 157-64.

[6]. Rand CS. Patient adherence with COPD therapy. Eur Respir Rev 2005; 96: 97-101.

[7]. Vestbo J, Anderson JA, Calverley PMA, Celli B, Ferguson GT, Jenkins C et al. Adherence to inhaled therapy, mortality and hospital admission in COPD. Thorax 2009;64: 939-43.

[8]. Bourbeau J, Bartlett SJ. Patient adherence in COPD. Thorax 2008; 63: 831-8.

[9]. Bischoff EWMA, Hamd DH, Sedeno M, Benedetti A, Schermer TRJ, Bernard S et al. Effects of a written action plan adherance on COPD exacerbation recovery. Thorax 2011; 66: 26-31. 